\title{
A history of diffuse large B cell lymphoma: aiming for better outcomes and higher cure rates
}

\section{Mini review}

Many accomplishments and progress have been made since 1958 when the Rappaport Classification system for lymphomas was introduced. ${ }^{1}$ Back then it was thought that large cell tumors were not of lymphoid origin. At the time, treatment was mainly palliative and consisted of nitrogen mustard and anti-metabolites combined with primitive radiation therapy. The 1980's were marked by change with the introduction of a new classification for lymphomas based on clinical course of the disease plus the use of more complex combination therapies. The CHOP regimen appeared in the picture definitely changing the treatment landscape for Non-Hodgkin's Lymphomas (NHL); prolonging patients' lives and providing better quality of life. ${ }^{2}$ Nowadays the WHO classification with over 40 subtypes of Lymphomas exists and with it came a more focused and dedicated approach to clinical research and better drugs. ${ }^{3}$ It is difficult, but not impossible to surpass, the efficacy shown by monoclonal antibodies such as Rituximab in the treatment of NHL in combination with chemotherapy; but who would have thought 15 years ago that CHOP's efficacy would be rivaled and exceeded. In benefit of patients, focus will always be on striving for cure, and in Diffuse Large B Cell Lymphoma (DLBCL) a considerable amount of patients behave as such after 10 years of Chemotherapy plus Rituximab. ${ }^{4}$

Diffuse Large B Cell Lymphomas are the most common subtype of NHL comprising nearly $30 \%$ of all cases. ${ }^{5}$ They are aggressive in nature and if left untreated survival is limited to days or months. Treatment is largely dependent upon extension of the disease (limited or extended), which is based upon several factors but mainly based upon radiation therapy field criteria. This classification is independent of the disease stage which is determined by the Lugano Criteria and/or Ann Arbor staging. ${ }^{6}$ For patients with limited stage disease, treatment consists of 3 chemotherapy cycles with Rituximab plus Radiation Therapy or 6-8 cycles or Rituximab with chemotherapy minus Radiation. ${ }^{7}$ Both have similar survival rates but differ in toxicity. For those who receive Radiation Therapy, toxicity is mainly based on the location of the irradiated site and for those who only receive systemic treatment, the hematologic and cardiac toxicity is greater than those who use the combined regimen ${ }^{8}$ in either case, limited disease patients can achieve Overall Survival rates at 10years greater than $75 \%$ with current treatment options. ${ }^{4}$ For de novo patients with extended disease, Radiation Therapy is not an option and these patients are treated with Chemotherapy plus Rituximab. ${ }^{9}$

The question whether the combined treatment approach is superior to Chemotherapy plus Rituximab is yet to be answered since there have been no trials conducted yet including Rituximab compared to short course Chemotherapy with Radiation Therapy. However several studies indicate similar or superior efficacy with short duration Chemotherapy with Radiation Therapy compared to 6-8cycles of Chemotherapy. One study (GELA LNH 93-1), suggests that patients with limited disease could benefit from involved field Radiation treatment after completing CHOP. ${ }^{10}$ Patients with risk factors for recurrence are more likely to benefit from prolonged Chemotherapy

\author{
Volume I Issue 2 - 2015
}

\author{
Michael J Liberman \\ Department of Hematology/oncology, Roche Pharmaceuticals, \\ USA
}

Correspondence: Michael J Liberman, Department of Hematology/oncology, Roche Pharmaceuticals, USA, Tel +506 22981647,Email mjliberman@yahoo.com

Received: April 15, 20I5 | Published: June 18, 2015

than the combined approach. ${ }^{9}$ During the Rituximab era, studies such as the MInT Trial have shown that patients with limited disease and favorable prognostic features benefit from shortened therapy $(89 \%$ event-free survival and $98 \%$ overall survival at three years). ${ }^{11}$ When Radiation Therapy is used, the dose should range between 30-40Gy. ${ }^{12}$ Bulky disease should be treated with extended Chemotherapy with Rituximab and involved field Radiation Therapy. Radiation alone is never recommended even with localized, early stage disease as patients are likely to relapse. ${ }^{5}$

Advanced DLBCL cases account for $70 \%$ of patients and their management is based primarily on Rituximab plus CHOP or CHOP like regimens without Radiation therapy as these normally have extended disease (Ann Arbor III, IV or bulky disease) which does not allow for this treatment modality. No other chemotherapy regimen has offered better disease free survival or overall survival than CHOP so its use in fit patients is not questioned. The use of an abbreviated course of CHOP therapy given every 14days vs the standard 21days with Rituximab has shown no additional benefit but does add considerable toxicity. ${ }^{13}$ Several other combinations have been tried in substitution of CHOP for patients with DLBCL with no efficacy benefit obtained. Only one trial using R-ACVBP which incorporates Vindesine showed benefit in a subgroup of patients (non-germinal center B cell like) showing an event free survival increase of $14 \%$ at three years and $8 \%$ in overall survival. ${ }^{10}$

In more recent years, several new agents are proving that indeed RCHOP therapy can be improved upon. Bortezomib has shown that when added to RCHOP, it can increase the partial response (PR)/ complete response (CR) rate to $88 \%$ with a $75 \% \mathrm{CR} .{ }^{14}$ Lenalidomide in this same scenario achieved a $92 \%$ overall response rate and a CR rate of $86 \%$. Hematologic toxicity though, was elevated with severe neutropenia, leukopenia and thrombocytopenia. ${ }^{15}$ Another Lenalidomide trial showed an overall response rate of $92 \%$ and $80 \% \mathrm{CR}$, practically mirroring the results achieved in the previously mentioned trial. ${ }^{16}$ For early 2017, Ibrutinib (a small molecule BTK inhibitor) is expected to gain approval in the relapsed/refractory scenario and later on in first line treatment for DLBCL. Other molecules such as anti CD30, anti CD79 and anti CD19/CD3 antibodies are in development and are expected to read out in the upcoming years. In 2014, in an effort to enhance quality of life in patients with Non-Hodgkin's Lymphoma 
and reduce infusion times, Roche Pharmaceuticals developed a subcutaneous, fixed dose formulation of Rituximab which has been approved by the European Medicinal Agency (EMA). This allows for a patient to receive the monoclonal anti CD20 antibody in a period of 5 to 7 minutes compared to $90-270$ minutes with the IV formulation. ${ }^{17}$

The same pharmaceutical company has developed a glycoengineered type II, fully humanized monoclonal anti CD20 antibody (Gazyva ${ }^{\circledR}$, Obinutuzumab) which enhances antibody dependent cellular cytotoxicity and direct cell death while avoiding complement dependent cytotoxicity. This change leads to a different binding mechanism to the CD20 receptor and increased affinity. ${ }^{18}$ Clinically, this is manifested in a $61 \%$ risk reduction for progression (26.7 vs 15.2 months) compared to Rituximab in the Chronic Lymphocytic Leukemia scenario. ${ }^{19}$ For this reason the Food and Drug Administration (FDA) in the United States and the EMA approved its use in this indication. ${ }^{20,21}$ The drug is being developed for NonHodgkin's Lymphoma in several trials both in the relapsed/refractory setting and in first line DLBCL (GADOLIN and GOYA studies) among others. The preliminary results are expected to be presented in mid-2015.

For relapsed/refractory patients, the management normally includes chemotherapy with or without Rituximab with the purpose of inducing the patient for a possible hematopoietic cell transplantation. ${ }^{9}$ Nearly $60 \%$ of eligible transplant patients will respond to chemotherapy. If this is not achieved, then the treatment scenario shifts to a palliative setting. Normally, chemotherapy regimens vary from those used in first line treatment. These include, but are not limited to DHAP (dexamethasone, high dose cytarabine and cisplatin), ESHAP (etoposide, prednisolone, cytarabine and cisplatin) and GemOx (gemcitabine, oxaliplatin).

\section{Conclusion}

In conclusion, the management of Diffuse Large B Cell Lymphoma has evolved significantly in the past 15 years and improvements have led to the possibility of cure for a considerable percentage of patients with the coming of targeted therapies. The WHO classification of B cell malignancies led to enhancement and a more directed approach in the clinical investigation of new drugs for the disease. In the upcoming years, more of these will enter late stage development and the combination scenarios could possibly lead to a chemotherapy free treatment in the mid to long term future.

\section{Acknowledgements}

None.

\section{Conflict of interest}

The author declares no conflict of interest.

\section{References}

1. Garvin AJ, Simon R, Young RC, et al. The Rappaport classification of non-Hodgkin's lymphomas: a closer look using other proposed classifications. Semin Oncol. 1980;7(3):234-243.

2. http://rchop.cancertreatment.net/

3. Harris NL, Jaffe ES, Diebold J, et al. World health organization classification of neoplastic diseases of the hematopoietic and lymphoid tissues: report of the clinical advisory committee meeting Airlie house, Virginia, November 1997. J Clin Oncol. 1999;17(12):3835-3849.
4. Coiffier B, Feugier P, Mounier N, et al. Long-term results of the GELA study comparing R-CHOP and CHOP chemotherapy in older patients with diffuse large B-cell lymphoma show good survival in poor-risk patients. Blood. 2010;116 (12).

5. Jonathan WF, Richard IF. Diffuse large B-cell lymphoma:hematology/ oncolog. Clinics of North America. 2008;22(5):941-952.

6. Cheson BD. Staging and response assessment in lymphomas: the new Lugano classification. Chin Clin Oncol. 2015;4(1):5.

7. Miller TP. The limits of limited stage lymphoma. J Clin Oncol. 2004;22(15):2982.

8. Llanos M, Tabernero J, Brunet J, et al. CHOP chemotherapy of intermediate and high grade non-hodgkin's lymphoma. Acta Oncol. 1994;33(8):935-939.

9. http://www.nccn.org/professionals/physician_gls/pdf/nhl.pdf

10. Reyes F, Lepage E, Ganem G, et al. ACVBP versus CHOP plus radiotherapy for localized aggressive lymphoma. $N$ Engl $\mathrm{J}$ Med. 2005;352(12):1197-1205.

11. Pfreundschuh M, Trumper L, Osterborg A, et al. CHOP like chemotherapy plus rituximab versus CHOP like chemotherapy alone in young patients with good prognosis diffuse large B cell lymphoma: a randomized controlled trial by the MabThera international trial (MInT) group. Lancet Oncol. 2006;7(5):379-391.

12. Ballonoff A, Rusthoven KE, Schwer A, et al. Outcomes and effect of radiotherapy in patients with stage I or II diffuse large B cell lymphoma: a surveillance, epidemiology, and end results analysis. Int J Radiat Oncol Biol Phys. 2008;72(5):1465-1471.

13. Watanabe T, Tobinai K, Shibata T, et al. Phase II/III study of R-CHOP-21 versus R-CHOP-14 for untreated indolent B-cell Non-hodgkin's lymphoma: JCOG 0203 trial. J Clin Oncol. 2011;29(30):3990-3998.

14. Ruan J, Martin P, Furman RR, et al. Bortezomib plus CHOP rituximab for previously untreated diffuse large B cell lymphoma and mantle cell lymphoma. J Clin Oncol. 2011;29(6):690-697.

15. Vitolo U, Chiappella A, Franceschetti S, et al. Lenalidomide plus RCHOP21 in elderly patients with untreated diffuse large lymphoma: results of the REAL07 open label, multicenter, phase 2 trial. Lancet Oncol. 2014;15(7):730-737.

16. Nowakowski GS, La Plant B, Macon WR, et al. Lenalidomide combined with RCHOP overcomes negative prognostic impact of nongerminal center phenotype in newly diagnosed diffuse large B cell lymphoma: a phase II study. J Clin Oncol. 2015;33(3):251-257.

17. http://www.ema.europa.eu/docs/en_GB/document_library/EPAR_ product_information/human/000165/WC500025821.pdf

18. http://www.ema.europa.eu/docs/en_GB/document_library/EPAR product_information/human/002799/WC500171594.pdf

19. Goede V, Fischer K, Busch R, et al. Obinutuzumab plus chlorambucil in patients with CLL and coexisting conditions. $N$ Engl J Med. 2014;370(12):1101-1110.

20. http://www.fda.gov/Drugs/InformationOnDrugs/ApprovedDrugs/ ucm373263.htm

21. http://www.ema.europa.eu/docs/en_GB/document_library/EPAR Product_Information/human/002799/WC500171594.pdf 